\title{
乌ु \\ Emittance compensation in split photoinjectors
}

\author{
Klaus Floettmann" \\ DESY, Notkestrasse 85, 22603 Hamburg, Germany
}

(Received 4 October 2016; published 11 January 2017)

\begin{abstract}
The compensation of correlated emittance contributions is of primary importance to optimize the performance of high brightness photoinjectors. While only extended numerical simulations can capture the complex beam dynamics of space-charge-dominated beams in sufficient detail to optimize a specific injector layout, simplified models are required to gain a deeper understanding of the involved dynamics, to guide the optimization procedure, and to interpret experimental results. In this paper, a slice envelope model for the emittance compensation process in a split photoinjector is presented. The emittance term is included in the analytical solution of the beam envelope in a drift, which is essential to take the emittance contribution due to a beam size mismatch into account. The appearance of two emittance minima in the drift is explained, and the matching into the booster cavity is discussed. A comparison with simulation results points out effects which are not treated in the envelope model, such as overfocusing and field nonlinearities.
\end{abstract}

DOI: 10.1103/PhysRevAccelBeams.20.013401

\section{INTRODUCTION}

Shortly after the invention of photocathode rf guns [1,2], the compensation of a correlated emittance contribution by a simple focusing scheme was reported by Carlsten [3]. The origin of the observed emittance contribution was identified in simulations to be the variation of the transversely defocusing space charge force with the longitudinal position within the bunch, as it appears in short bunches with a relatively high charge. Carlsten discussed the compensation process by considering longitudinal slices of the bunch, which spread out in phase space as a result of the varying space charge force. Assuming a development of the beam radius $r$ as $r(z)=r_{0}\left(1+b z^{2}\right)$, where $b$ describes the local defocusing strength of the space charge field $\left(r_{0}\right.$ is the initial radius and $z$ the longitudinal coordinate), he could show that the projected emittance increases in a drift and reduces again after a focusing lens. Characteristic features of the emittance development in the analytical theory are an emittance maximum coinciding with the maximum beam size and two emittance minima in the vicinity of the beam size minimum in the drift behind the lens. These features can also be found in simulations (e.g., [4]) and experiments [5] and are not explained by other models describing the emittance compensation process.

While the emittance development in Carlsten's model essentially takes place in drift sections (the emittance stays

\section{*Klaus.Floettmann@DESY.De}

Published by the American Physical Society under the terms of the Creative Commons Attribution 4.0 International license. Further distribution of this work must maintain attribution to the author(s) and the published article's title, journal citation, and DOI. constant in the thin focusing lens), the emittance compensation theory developed by Serafini and Rosenzweig [6] starts by considering a beam in a constant focusing channel. The Brillouin flow in a focusing channel is characterized by a beam traveling with a constant beam size, which requires that the defocusing space charge force is compensated by the external focusing force. Small excursions around the matched beam condition lead to an oscillation of the beam envelope around the equilibrium beam size. Since the period length of the oscillation turns out to depend only on the strength of the external focusing force and not on the strength of the defocusing space charge force, a periodic emittance oscillation appears. Based on this result, the theory is extended to the case of a combined focusing and acceleration channel. Instead of a constant equilibrium beam size, the beam needs to be matched to the so-called invariant envelope in a combined channel. Alternative derivations of the invariant envelope theory are presented in Ref. [7], while an extension of the theory to elliptical beams is discussed in Ref. [8].

In this paper, an analytic solution of the envelope equation in a drift with space charge is derived as a basis for the discussion of the emittance compensation process. The analytic solution includes the emittance term, which is not the case in the previously developed models. The discussion is intended to describe the emittance development in a so-called split photoinjector, i.e., an injector where the short rf gun (typically, $1 \frac{1}{2}$ cells) is followed by a drift in which the beam is focused before it is matched into a booster cavity to increase the energy and to freeze out the emittance development. The appearance of two emittance minima in the vicinity of the beam focus in the drift is explained by considering the beam envelopes of two slices of a bunch. A brief discussion of the invariant envelope theory leads to the conclusion that the matching into the 
booster cavity which yields the minimal emittance in a split photoinjector does not meet the invariant envelope conditions. In the final section, simulation results are presented and compared to the analytical model. Important aspects which are not captured by the slice envelope model, such as the generation of a beam halo by overfocused particles and the slice emittance growth due to field nonlinearities near the photocathode, are highlighted.

\section{SPACE CHARGE FIELD OF A BUNCHED BEAM}

Throughout the paper, cylindrical symmetry of all fields is assumed. General characteristics of the space charge field of rotational symmetric bunched beams can be illustrated at the example of a homogeneously charged cylinder of length $L$, radius $R$, and aspect ratio $A=R / L$.

The longitudinal on-axis electric field component can be calculated by direct integration as

$$
E_{z}(\zeta, A)=E_{z}^{\perp} H(\zeta, A),
$$

where $E_{z}^{\perp}=\frac{Q}{2 \pi \epsilon_{0} R^{2}}$ is the field of an infinitesimal thin sheet of charge $Q . \epsilon_{0}$ is the vacuum permittivity, and the function $H(\zeta, A)$ is given by

$$
\begin{aligned}
H(\zeta, A)= & \sqrt{\left(\frac{1}{2}-\frac{\zeta}{L}\right)^{2}+A^{2}}-\sqrt{\left(\frac{1}{2}+\frac{\zeta}{L}\right)^{2}+A^{2}} \\
& -\left|\frac{1}{2}-\frac{\zeta}{L}\right|+\left|\frac{1}{2}+\frac{\zeta}{L}\right| .
\end{aligned}
$$

$\zeta$ is the relative longitudinal position in the bunch; i.e., $\zeta=0$ is the center of the bunch.

Equation (1) describes the electric field in the rest frame of a bunch; therefore, the bunch length is elongated by the Lorentz factor $\gamma$ as compared to the laboratory frame, i.e., $L=\gamma L_{\text {lab }}$. The aspect ratio shrinks hence with increasing beam energy, while $H$ converges to zero. For very short bunches, i.e., $A>1, H$ describes within the boundaries of the bunch a linear relation between -1 (at $\zeta / L=-0.5$ ) and +1 (at $\zeta / L=0.5$ ). The longitudinal field of a short bunch is hence described by the field of an infinitesimal thin sheet of charge, which is reached at the ends of the charge distribution and a linear interpolation in between the ends.

Figure 1 (top) displays the linear depends for large aspect ratios. The lowering of $H$ for small aspect ratios is accompanied by the development of nonlinearities at the ends of the bunch.

The corresponding radial field can be calculated in linear approximation by [9]

$$
E_{r}(r, \zeta, A)=E_{r}^{l}(r) G(\zeta, A),
$$

where $E_{r}^{l}(r)=\frac{Q r}{2 \pi \epsilon_{0} R^{2} L},(r<R)$ is the radial electric field inside an infinitely long cylindrical charge distribution, and the function $G(\zeta, A)$ is given by
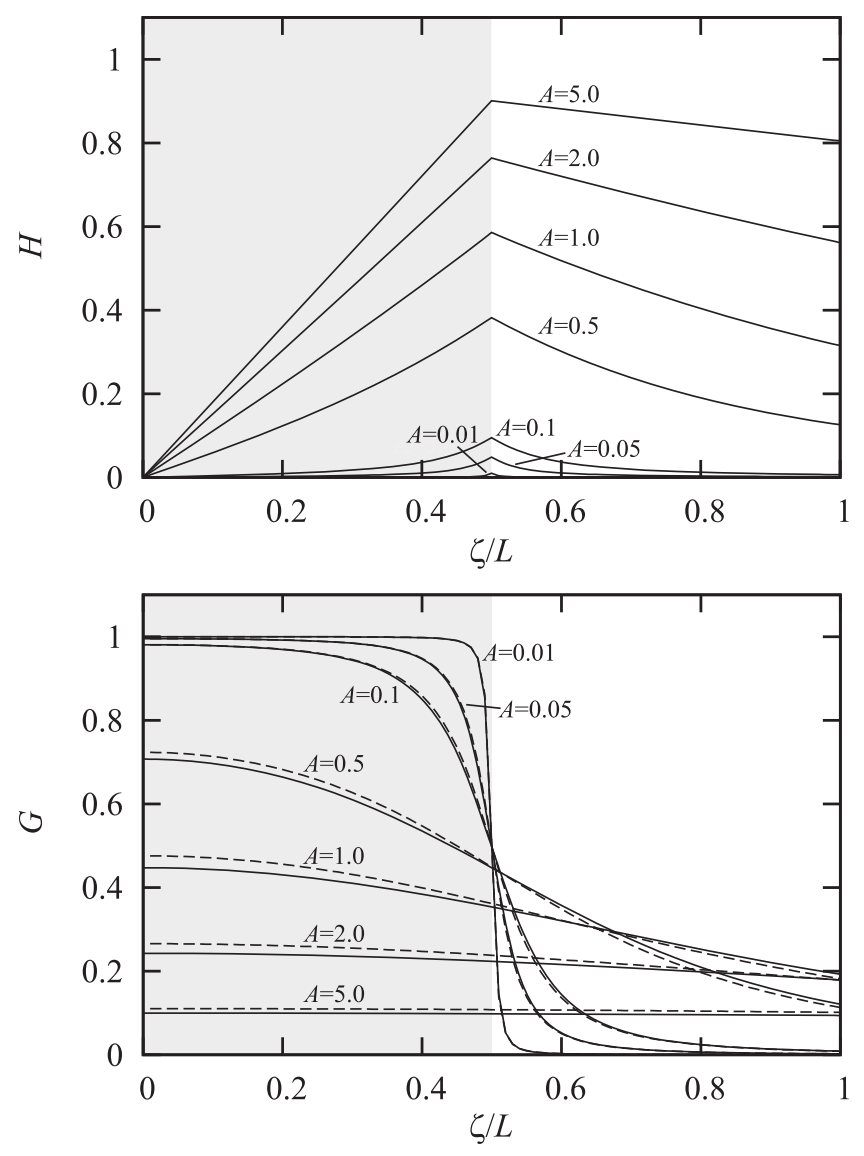

FIG. 1. The function $H$ [top, Eq. (2)] and $G$ [bottom, Eq. (4)] for various aspect ratios $A$ and positive values of $\zeta / L$. $H$ is antisymmetric and $G$ is symmetric to the origin. The bunch extends up to $\zeta / L=0.5$ as indicated by the gray shading. Broken lines in the lower plot show the results of a numerical integration at a radius $r=R / 2$.

$G(\zeta, A)=\frac{1}{2}\left[\frac{\frac{1}{2}-\frac{\zeta}{L}}{\sqrt{\left(\frac{1}{2}-\frac{\zeta}{L}\right)^{2}+A^{2}}}+\frac{\frac{1}{2}+\frac{\zeta}{L}}{\sqrt{\left(\frac{1}{2}+\frac{\zeta}{L}\right)^{2}+A^{2}}}\right]$

Figure 1 (bottom) shows a comparison of $G$ with numerical results for various aspect ratios $A$. For the numerical results, the rms beam radius $r=R / 2$ is chosen. The uniformity of $G$ for very small aspect ratios $A \leq 0.01$ reflects the uniformity of the charge density in this example. For aspect ratios between $\sim 0.05$ and $\sim 1.0$, the radial field can vary substantially within the bunch, while it is more or less constant for very small and for very large aspect ratios. For large aspect ratios, the field amplitude shrinks and tends to zero for very short bunches. $[G(\zeta=0, A) \approx 1 /(2 A)$ for $A>1$.] However, not only the field amplitude shrinks for large aspect ratios, but the radial field becomes also increasingly nonlinear as shown in Fig. 2, where the radial electric field at $\zeta=0$ is plotted as a function of the radial position. The curves in the plot are normalized such that at the rms radius a field value 


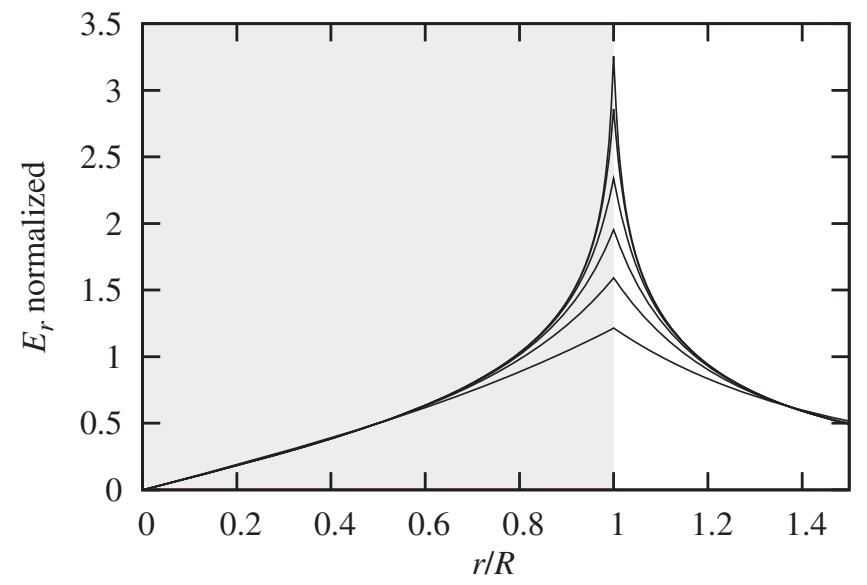

FIG. 2. Normalized radial electric field versus the radius in the center of the bunch $(\zeta=0)$. The aspect ratio for the most linear curve is $A=1.0$, followed by $A=(2.5,5.0,10.0,25.0,50.0)$.

of 0.5 is reached. Equation (3) matches within the accuracy shown in Fig. 1 to this point and can therefore be used to describe the evolution of the rms beam envelope. The emittance growth introduced by the radial nonlinearity of the fields will, however, be missed.

The discussion above applies to the beam in its average rest frame. In terms of the bunch length in the laboratory frame $L_{\text {lab }}$ the aspect ratio reads hence as $A=R /\left(\gamma L_{\mathrm{lab}}\right)$. Upon transformation into the laboratory frame, the transverse electric field needs to be multiplied by $\gamma$. In addition, the corresponding azimuthal magnetic field $B_{\theta}=\beta \gamma E_{r} / c$ needs to be taken into account. Here $c$ is the velocity of light, $\beta c$ is the velocity which describes the Lorentz transform from the average rest system into the laboratory system, $\gamma$ is the corresponding Lorentz factor, and $E_{r}$ is the radial electric field in the rest system.

The transverse force due to the space charge field is hence given as

$$
F_{r}=\frac{e Q}{2 \pi \epsilon_{0} L_{\mathrm{lab}} \gamma^{2}} \frac{r}{R^{2}} G(\zeta, A)
$$

Transferring to Cartesian coordinates, the differential transverse envelope equation in a drift with space charge follows as

$$
\sigma^{\prime \prime}-\frac{P}{4 \sigma} G(\zeta, A)-\frac{\varepsilon^{2}}{\sigma^{3}}=0,
$$

where the transverse rms beam size and its second derivative with respect to the longitudinal coordinate $z$ are denoted as $\sigma$ and $\sigma^{\prime \prime}$, respectively, $R$ is replaced by $2 \sigma$, the geometrical beam emittance is denoted by $\varepsilon$, and $P$ stands for the generalized perveance of the beam:

$$
\begin{aligned}
P & =\frac{e Q}{2 \pi \epsilon_{0} m_{e} c^{2} L_{\mathrm{lab}} \beta^{2} \gamma^{3}} \\
& =\frac{2 I}{I_{A} \beta^{3} \gamma^{3}} .
\end{aligned}
$$

Here the average beam current $I=\frac{Q c \beta}{L_{\mathrm{lab}}}$ and the Alfvén current $I_{A}=\frac{4 \pi \epsilon_{0} m_{e} c^{3}}{e} \simeq 17 \mathrm{kA}$ are introduced.

The envelope equation is valid only if the emittance is a constant of motion. Thus, nonlinear terms of the space charge force are ignored, and the generalized perveance describes the linear defocusing space charge term only. The dependence of $G$ on the aspect ratio implies an additional dependence of the envelope equation on the beam energy but also on the bunch length and on the transverse beam size. While the first is relatively constant in an injector except very near to the cathode, the latter changes significantly. This implicit dependence cannot be treated; i.e., $G$ is assumed to be constant.

Moreover, $G$ depends on the nonstationary charge distribution itself and thus develops under the influence of the space charge field and the beam emittance, which is also ignored in the analytical treatment. Because, even if a perfect cylindrical distribution is launched at the cathode of an rf gun, it will be only approximately cylindrical at the exit of the gun, and thus $G$ will be different. Figures 1 and 2 illustrate the fields in a bunched beam only, for a realistic distribution; e.g., in the drift behind an rf gun, significant deviations are to be expected.

A full treatment of the space-charge-dominated dynamics requires numerical simulations. The aim of the analytic discussion below is to develop a simple model which explains characteristic features and helps to interpret numerical results. We will thus consider decoupled envelope equations of two slices of a bunch with a fixed perveance only. The difference in the development of the two slices-one representing the central part of the bunch, the other a slice in the head or the tail-leads to correlated emittance contributions which can vanish again under certain circumstances. Instead of the complex dynamics in the rf gun, only a simple drift with a fixed beam energy will be considered. This rigorous simplification is possible because the basic correlation which builds up in phase space is driven by the difference of the defocusing space charge term and thus builds up in a drift space as well.

\section{TRANSVERSE ENVELOPE EQUATION IN A DRIFT WITH SPACE CHARGE}

Equation (6) has no simple analytic solution, but a solution in the form of an infinite polynomial series can be formulated. The derivation is simplified by setting $z=0$ at the position of a focus, where the beam size is denoted as $\sigma_{0}$. Since the emittance is conserved, the beam envelope needs to be symmetric with respect to the focus position and thus follows the form 


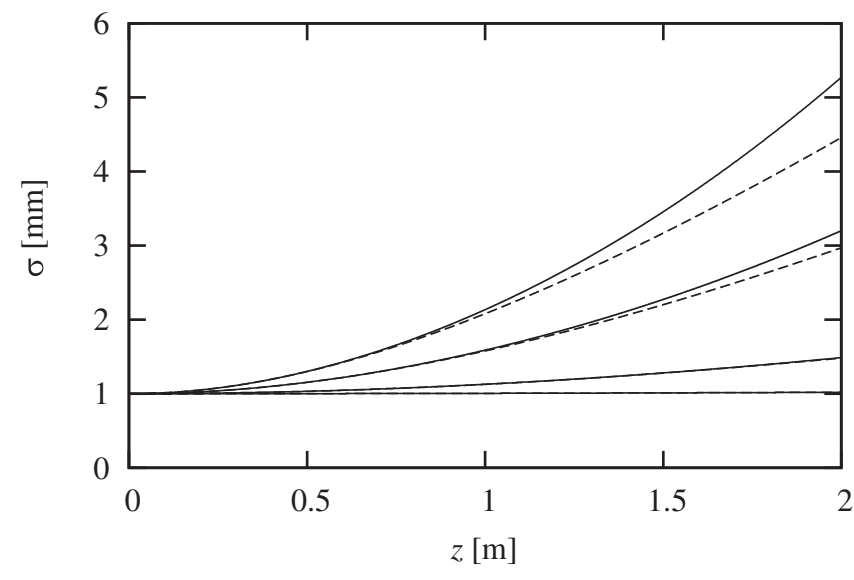

FIG. 3. Development of the beam size in a drift with space charge. Beam parameters: $\gamma=10, \quad \varepsilon_{n} / \gamma=10^{-7}, \quad P=$ $(10,5,1,0) \times 10^{-6}$ (the highest perveance corresponds to the highest beam divergence). Analytical approximation according to Eq. (8) with coefficients $c, \ldots=0$ (solid lines) and results of a numerical integration of Eq. (6) (broken lines).

$$
\sigma=\left(\sigma_{0}^{2}+a z^{2}+b z^{4}+c z^{6} \ldots\right)^{1 / 2} .
$$

The coefficients $a, b, c, \ldots$ need to be determined. For vanishing space charge forces, Eq. (8) has to converge to the known solution without space charge, i.e., to $a=\frac{\varepsilon^{2}}{\sigma_{0}^{2}}$ and $b, c, \ldots=0$.

In the following, this ansatz is followed only up to the fourth order in $z$, i.e., coefficient $b$. This yields a good description of the beam envelope near a focus and a still reasonable approximation in larger distance to the focus. For a discussion of the convergence properties of the polynomial solution, see the Appendix.

Introducing Eq. (8) into Eq. (6) leads to $[G(\zeta, A)=1]$

$$
\begin{aligned}
& \sigma_{0}^{2}\left(a-\frac{P}{4}\right)+\left(6 b \sigma_{0}^{2}-a \frac{P}{4}\right) z^{2} \\
& \quad+\left(3 a-\frac{P}{4}\right) b z^{4}+2 b^{2} z^{6}=\varepsilon^{2} .
\end{aligned}
$$

Equation (9) is fulfilled at $z=0$ by setting

$$
a=\frac{\varepsilon^{2}}{\sigma_{0}^{2}}+\frac{P}{4} .
$$

As required, $a$ converges to $\frac{\varepsilon^{2}}{\sigma_{0}^{2}}$ for negligible space charge, while it converges for $P \gg \varepsilon$ to $P / 4$ as proposed by Harrison [10] as the approximate value to describe the envelope of a space-charge-dominated beam near the focus.

Ignoring terms proportional to $z^{4}$ and higher, we find furthermore

$$
b=\frac{a P}{24 \sigma_{0}^{2}} .
$$

In Fig. 3, the analytic approximation up to fourth order in $z$ is compared to a numerical integration of the envelope equation. The highest generalized perveance of $10^{-5}$ corresponds to a beam current of $\sim 85 \mathrm{~A}$ at $\gamma=10$ and is hence comparable to the beam conditions in the drift behind a $1 \frac{1}{2}$ cell rf gun. Note that here $\frac{\varepsilon^{2}}{\sigma_{0}^{2}} \ll P / 4$ holds in all but the $P=0$ case.

The series approximation is not convergent; i.e., when the beam size becomes significantly larger than the size at the focus, an increasing number of coefficients needs to be taken into account to improve the accuracy. In the vicinity of a focus, however, a good agreement is reached even in the case of a strong space charge contribution.

\section{CORRELATED EMITTANCE GROWTH AND ITS COMPENSATION IN A DRIFT}

The space charge dependence of the beam size as shown in Fig. 3 and the dependence of the space charge force of the longitudinal position inside a bunch as illustrated by Fig. 1 (plus additional variations of the space charge force due to a nonuniform current or due to energy variations within the bunch) indicate a general source for a correlated emittance growth.

For a discussion of this emittance growth and its compensation, we consider two slices of a bunch, one traveling under the influence of a high perveance, thus representing the central part of the bunch, and a second one traveling under the influence of a lower perveance, thus representing a part in the head or the tail of the bunch. For the examples shown in the following plots, the same parameters as used for Fig. 3 with the high perveance $P_{h}=10^{-5}$ and the low perveance $P_{l}=5 \times 10^{-6}$ are used, thus spanning a range of a factor of 2 in the perveance. Each slice has an associated slice emittance (of equal value). The emittance of the beam is then formed by a superposition of the two slices with their respective orientation and form in phase space. In the initial condition, the slices overlap with $\sigma^{\prime}=0$.

In order to understand the emittance growth, we need to know the orientation of the rms ellipse in phase space, in addition to the slice size. The slope $m$ of the ellipse follows from a straight line fit to the phase space distribution:

$$
m=\frac{\left\langle x x^{\prime}\right\rangle}{\left\langle x^{2}\right\rangle}
$$

where \langle\rangle defines an ensemble average, $x$ and $x^{\prime}$ represent phase space coordinates, and $\langle x\rangle=\left\langle x^{\prime}\right\rangle=0$ is assumed. The slope can be related to the derivative of the beam envelope with respect to $z$ by

$$
\sigma^{\prime}=\frac{\partial}{\partial z}\left\langle x^{2}\right\rangle=\frac{\left\langle x x^{\prime}\right\rangle}{\left\langle x^{2}\right\rangle^{1 / 2}} .
$$




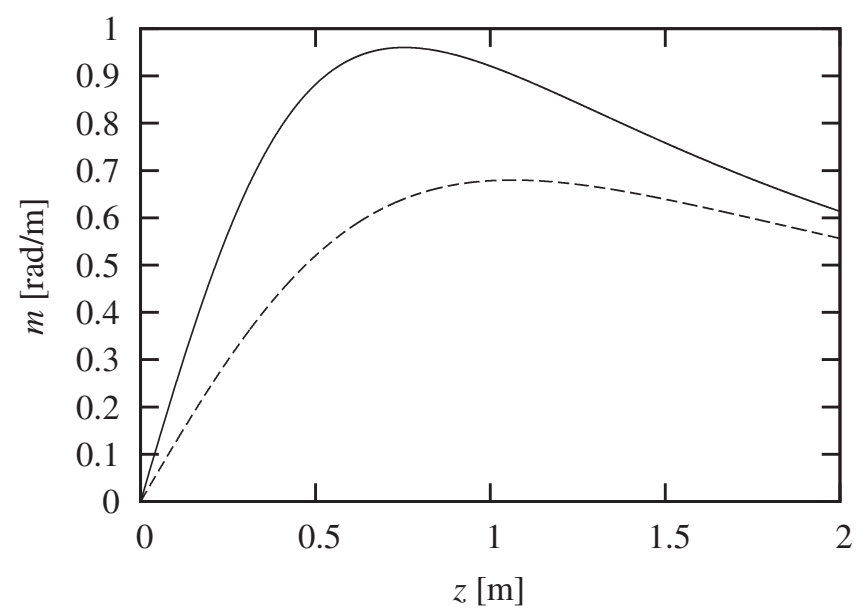

FIG. 4. Development of the slopes of the phase space ellipses corresponding to Fig. 3 (only numerical results) for the case with $P=10^{-5}$ (solid line) and $P=5 \times 10^{-6}$ (broken line). Close to the maxima of the slopes, also the difference between the slopes is maximal. Further downstream, the spread reduces again.

Thus,

$$
m=\frac{\left\langle x x^{\prime}\right\rangle}{\left\langle x^{2}\right\rangle}=\frac{\sigma^{\prime}}{\sigma}
$$

Right after the focus, $\sigma^{\prime}=\frac{1}{2 \sigma}\left(2 a z+4 b z^{3}+\cdots\right)$ increases rapidly, while the beam size increases only moderately. $m$ increases, hence, and a fanlike structure opens in phase space due to the variation of the perveance. Further downstream, however, $\sigma$ increases faster than $\sigma^{\prime}$, and $m$ decreases again. Also, as demonstrated in Fig. 4, the spread of the slopes shrinks, because the beam size of the high perveance slice is growing faster than the beam size of the low perveance slice. Thus, the fanlike structure in the phase space closes again. The closing of a fanlike structure in phase space in a drift is not unique to the case under discussion here but also appears in the case of a time-dependent focusing; see the discussion in Ref. [12] (Sec. XIII) for details.

It is instructive to discuss the case where the term proportional to $z^{4}$ in Eq. (8) is ignored in the development of the slice envelope. Indicating the parameters for the high perveance slice with index $h$ and the parameters for the low perveance with index $l$, the ratio of the slopes reads as

$$
\frac{\sigma_{l} \sigma_{h}}{\sigma_{l} \sigma_{h^{\prime}}}=\frac{\sigma_{0}^{2} a_{l} z+a_{l} a_{h} z^{3}}{\sigma_{0}^{2} a_{h} z+a_{l} a_{h} z^{3}} .
$$

Equation (15) converges to 1 for large $z$; i.e., in this limiting case, the two ellipses have the same slope and the emittance growth is totally determined by the mismatch of the beam sizes as indicated in Fig. 5.

The emittance for a pure beam size mismatch is given by

$$
\varepsilon=\varepsilon_{s} \frac{\sigma_{l}^{\prime 2}+\sigma_{h}{ }^{2}}{2 \sigma_{l} \sigma_{h}} \simeq \varepsilon_{s} \frac{a_{l}+a_{h}}{2 \sqrt{a_{l} a_{h}}}
$$

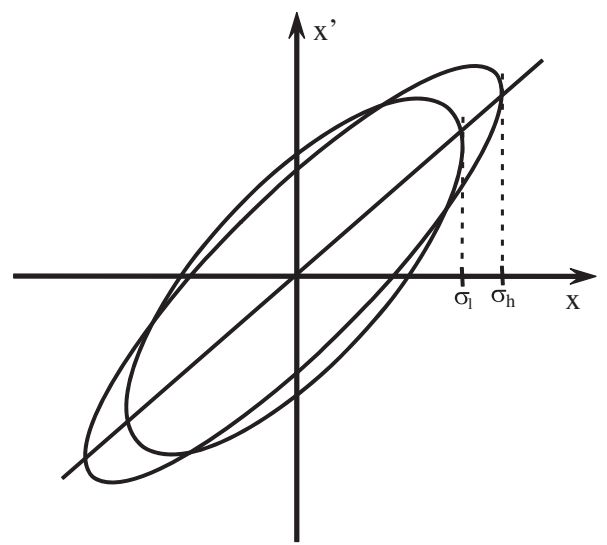

FIG. 5. Emittance growth due to a mismatch of the beam sizes.

where $\varepsilon_{s}$ denotes the slice emittance. The approximation in the second step applies for large $z$ to the case discussed above.

If we now reverse the development of one slice envelope by applying a focusing kick which transfers $\sigma^{\prime}$ to $-\sigma^{\prime}$ and let the beam drift again, the same focusing kick of a linear focusing element will reverse the development of the other slice envelope exactly, and both slices will reach a focus with the same slice size at the same longitudinal position. The emittance growth is hence completely reversible in this limiting case where $m$ is equal for the two slices.

If $m$ is not equal, it is not possible to reverse the development of both slices exactly with a linear focusing element as depicted in Fig. 6. For this numerical result, a linear focusing kick is applied to two slices after $2 \mathrm{~m}$ initial drift as shown in Fig. 3. The low perveance slice reaches a lower focus earlier in the development than the high

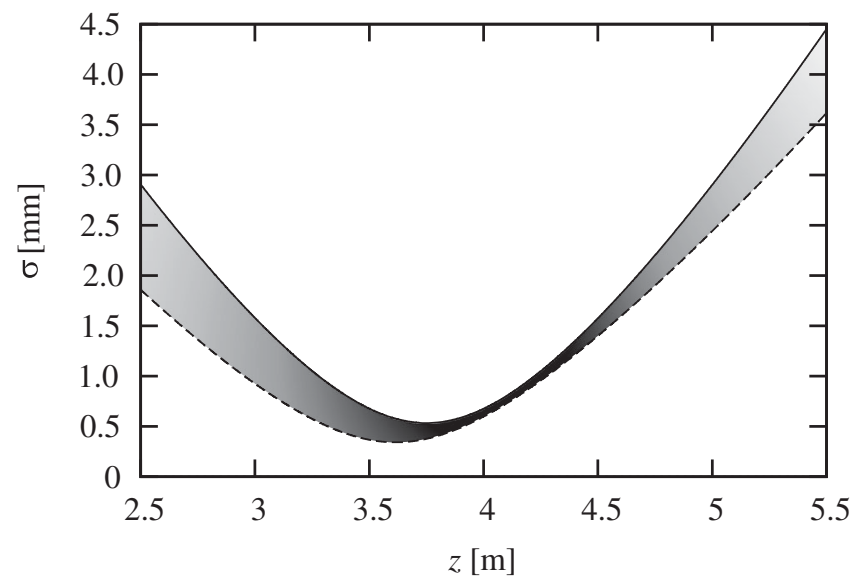

FIG. 6. Slice size development of the high perveance slice (solid line) and the low perveance slice (broken line) after a focusing kick from a numerical integration of the envelope equation. The development within the first $2 \mathrm{~m}$ is shown in Fig. 3; the focusing kick is applied at $z=2 \mathrm{~m}$. The envelopes of slices with intermediate perveance will fall into the gray shaded band. 
perveance slice. For the development of the beam emittance, the relative orientation of the two ellipses is again of basic significance. In the case of equal orientation, the condition

$$
\frac{\sigma_{l}^{\prime}}{\sigma_{l}}-\frac{\sigma_{h}^{\prime}}{\sigma_{h}}=0
$$

holds. Since we are interested in the conditions in the vicinity of the focus, we can again ignore the higher orders term in $z$ in the analytic approximation. Equation (17) takes hence the form

$\sigma_{h, 0}^{2} a_{l} \Delta z_{l}-\sigma_{l, 0}^{2} a_{h} \Delta z_{h}+a_{l} a_{h}\left(\Delta z_{h}^{2} \Delta z_{l}-\Delta z_{l}^{2} \Delta z_{h}\right)=0$,

where the minimal slice sizes occurring at the positions $z_{h}$ and $z_{l}$ are denoted as $\sigma_{h, 0}$ and $\sigma_{l, 0}$, respectively, and the relative distances to the location of the minima are denoted as $\Delta z_{h}=z-z_{h}$ and $\Delta z_{l}=z-z_{l}$.

Expansion of $\Delta z_{l}^{2} \Delta z_{h}$ yields

$\Delta z_{l}^{2} \Delta z_{h}=z^{3}-2 z^{2} z_{l}+z_{l}^{2} z-z^{2} z_{h}+2 z^{2} z_{l} z_{h}-z_{l}^{2} z_{h}$.

For the equivalent expansion of $\Delta z_{h}^{2} \Delta z_{l}$, indices $h$ and $l$ need to be exchanged.

The interest in this derivation lies in the fact that the term proportional $z^{3}$ cancels. Equation (17) is hence a quadratic equation in $z$ and thus has two solutions. This leads to two emittance minima in the vicinity of the average focus, as will be shown in the following.

Figure 7 shows the development of the slopes of the phase space ellipses corresponding to the envelope development shown in Fig. 6. Near the focus, the two lines cross

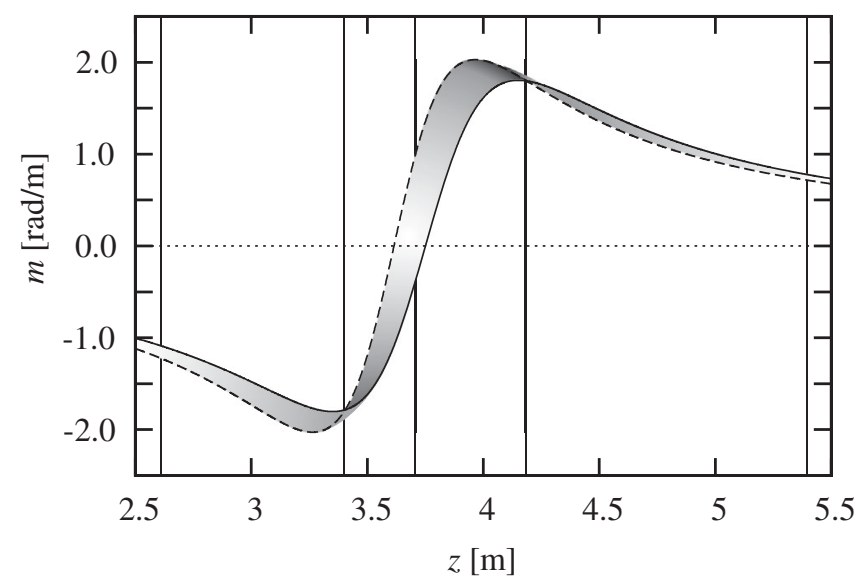

FIG. 7. Development of the slopes of the phase space ellipses for the high perveance slice (solid line) and the low perveance slice (broken line). The plot corresponds to Fig. 6. The solutions for slices with intermediate perveance are indicated by the gray shaded band. Vertical lines at $z=2.6,3.4,3.7,4.2$, and $5.4 \mathrm{~m}$ indicate the positions referred to in Fig. 8. at two points as expected from the derivation above. Solutions for intermediate perveance will not cross at exactly the same points, but in close vicinity, so that two emittance minima appear which correspond to aligned phase space ellipses with a mismatch of the beam size.

In Fig. 8, the beam divergence is plotted as a function of the beam size. Connecting lines which correspond to the longitudinal positions marked in Fig. 7 indicate the relative alignment of the phase space ellipses. At two points, the connecting lines point toward the origin of the coordinate system; i.e., the ellipses are aligned.

Figure 9 presents the development of the beam emittance as it is derived from the development of the slice envelopes shown in Fig. 6. In addition to the standard emittance calculation, a calculation which ignores the individual slice emittance is plotted. This reaches, in coincidence with Figs. 7 and 8, a zero emittance at the locations of equal orientation of the phase space lines. The real emittance at these points is determined by a beam size mismatch, which is larger at the location of the first minimum as visible in Fig. 6. In cases of large slice emittance and/or large beam size mismatch, this first emittance minimum can be strongly washed out, so that only one emittance minimum becomes evident. (Also, crossover particles, which will be discussed in Sec. VI, contribute more to the emittance at the first minimum than to the emittance in the second minimum.)

The longitudinal distance of the two emittance minima as well as the strength of the emittance oscillation depends on the difference of the perveance in the two slices under

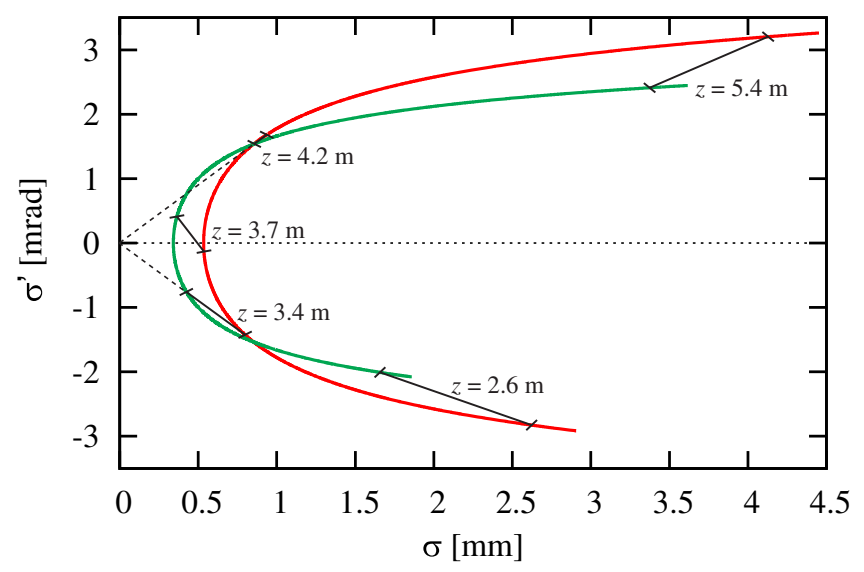

FIG. 8. Divergence versus beam size during the emittance compensation process. The high perveance slice (red curve) exhibits greater excursions in beam size and divergence than the low perveance slice (green curve). Black lines connect points which correspond to the same longitudinal position-marked in Fig. 7 - and thus indicate the development in time. At $z=3.4 \mathrm{~m}$ and $z=4.2 \mathrm{~m}$, the extensions of the connecting lines (broken) cross the origin of the coordinate system; i.e., the slices are aligned. The shorter length of the connecting line at $z=4.2 \mathrm{~m}$ corresponds to a smaller emittance contribution due to the beam size mismatch. 


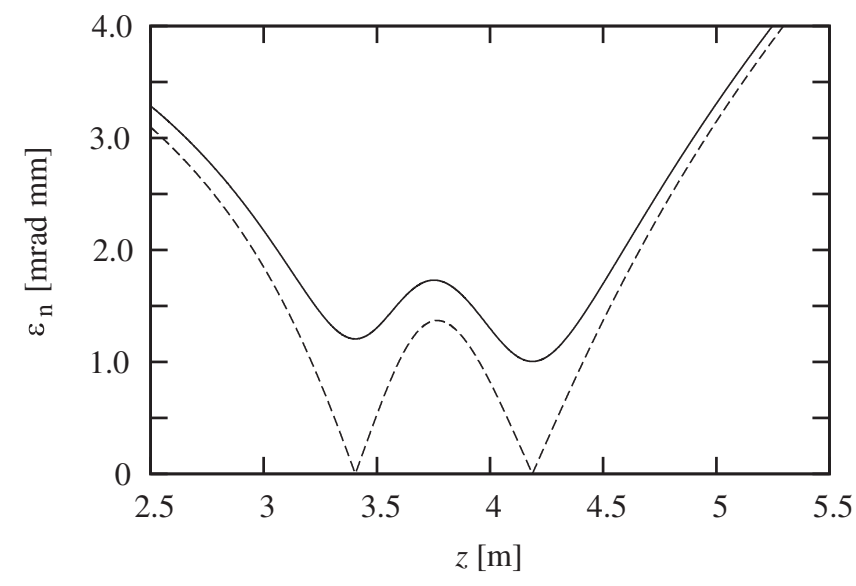

FIG. 9. Development of the beam emittance as calculated from the high perveance and low perveance slices. The broken line ignores the slice emittance; i.e., the slices are treated as lines in phase space. The emittance minima coincide with the locations of equal slopes as shown in Fig. 7.

consideration. For small differences, the oscillation is suppressed and the minima shift closer together, so that also in this case only one shallow emittance minimum becomes evident.

In the beginning of this section, the reversibility of the slice size development was discussed. With a realistic space charge model, the development of the individual slice sizes turns out to be irreversible, if only a linear focusing element is applied. However, the development of the average beam size is, of course, still reversible, which can be used to set a scale for the applied focusing strength. Compared to this average reversibility condition, a $15 \%$ stronger focusing kick is applied in the calculations for Figs. 6-9. This reduces the beam size mismatch for the second emittance minimum by shifting the beam envelopes in this area closer together, while the mismatch for the first minimum is increased. With even stronger focusing kicks, the mismatch can be further minimized, and the envelopes can even cross in the vicinity of the second minimum. For focusing kicks lower than the average reversibility condition, the mismatch for the first minimum is reduced and the location of the second minimum shifts far to the right. The lower focus appearing earlier in the development of the low perveance slice favors, however, the second minimum, and a stronger focusing kick is, in general, preferable.

A realistic bunch consists, of course, not of two slices only but may be considered as composed of a number of slices with different perveance. If additional slices with intermediate perveance are included in our calculation, they will fill the area between the two lines in Fig. 6; the lines will, however, not all cross at the same locations in Fig. 7. Whether the emittance development as plotted in Fig. 9 is found for a realistic beam depends hence on numerous factors such as the total spread of the perveances in the beam and the slice emittance. Two emittance minima are typically found for bunches with a uniform longitudinal charge distribution, while often only one emittance minimum is found for beams with a Gaussian longitudinal charge distribution (large spread of the perveances and large slice emittance) or for ellipsoidal beams (small spread of the perveances).

For a beam starting from a cathode inside an rf gun, the required correlation between slice size and orientation in phase space at the entrance of the focusing element may not be as strict as in our simple model. The rf focusing, for example, leads to conditions where slices with the same perveance in the head and the tail of the bunch have a different orientation in phase space. Additionally, the perveance of a slice is determined not only by the charge distribution and the slice position within the bunch but also by the energy of the slice, which depends again on space charge effects but also on the rf phase. It is, hence, conceivable that a careful tuning of the rf phase can partially compensate other detrimental effects.

\section{EMITTANCE COMPENSATION IN FOCUSING CHANNELS}

In a split photoinjector, the emittance compensation process takes primarily place in the drift between the $\mathrm{rf}$ gun and the first accelerating section. In the subsequent accelerating section (booster cavity), the beam energy increases so that the space charge force is reduced [Eq. (5)]. In addition, the spread of the perveances is reduced due to the decreasing aspect ratio (in the rest frame) (Fig. 1). Besides the longitudinal accelerating force, the beam is influenced by a ponderomotive transverse focusing force in the booster cavity and possibly by the field of an external solenoid which may be wrapped around the accelerating structure.

Thus, the envelope equation needs to be extended by the so-called adiabatic damping term $\frac{\gamma^{\prime}}{\gamma} \sigma^{\prime}$ and a term describing the rf and the solenoid focusing. In order to take the energy dependence explicitly into account, we will moreover replace the perveance by $K_{s}=P \gamma^{3} / 4$ and the geometrical emittance by the normalized emittance divided by gamma, $\varepsilon=\varepsilon_{n} / \gamma$. For $\beta=1$, the differential envelope equation takes thus the form

$$
\sigma^{\prime \prime}+\frac{\gamma^{\prime}}{\gamma} \sigma^{\prime}+\frac{K}{\gamma^{2}} \sigma-\frac{K_{s}}{\gamma^{3} \sigma}-\frac{\varepsilon_{n}^{2}}{\gamma^{2} \sigma^{3}}=0
$$

The term $\frac{\gamma^{\prime}}{\gamma} \sigma^{\prime}$ takes the reduction of the angular spread due to the growing longitudinal momentum into account; i.e., the transverse momentum does not change and the term does not describe a dissipation of energy as the notion "damping term" might suggest. $\gamma^{\prime}=\frac{\partial}{\partial z} \gamma$ is the average energy gain in terms of the rest energy of the electron. $K=\left(\frac{e B_{z}}{2 m_{e} c}\right)^{2}+\frac{\gamma^{\prime 2}}{8 \sin ^{2} \phi}$ sums up the focusing contribution of a solenoid with constant field $B_{z}$ (with the rest mass of the 
electron $m_{e}$ ) and the ponderomotive focusing term of the rf with the rf phase $\phi$ according to Ref. [13].

Equation (20) has no general solution, but some special solutions can be formulated. Particular interest exists in solutions where the defocusing and the focusing terms are balanced. The simplest case with $\gamma^{\prime}=0$, i.e., a solenoid section without acceleration, has been discussed by Brillouin [14,15]. The matched beam size is constant and follows from $\frac{K}{\gamma^{2}} \sigma=\frac{K_{s}}{\gamma^{3} \sigma}+\frac{\varepsilon_{n}^{2}}{\gamma^{2} \sigma^{3}}$ as

$$
\sigma_{m}^{2}=\frac{K_{s}}{2 K \gamma}+\sqrt{\left(\frac{K_{s}}{2 K \gamma}\right)^{2}+\frac{\varepsilon_{n}^{2}}{K}} .
$$

Small distortions $\Delta \sigma$ and $\Delta \sigma^{\prime}$ of the initial conditions lead to an oscillatory solution around the matched beam size of the form

$$
\sigma \simeq \sigma_{m}+\Delta \sigma \cos k z+\frac{1}{2} \Delta \sigma^{\prime} \sin k z
$$

with the wave number $k$ given by

$$
k=\frac{\sqrt{2 K}}{\gamma} \text {. }
$$

The wave number depends, hence, only on the external focusing fields and not on the defocusing perveance term. It is this property of the matched beam envelope which stimulated the interest in this class of solutions for focusing channels. Slices in a beam which is matched to the average perveance will oscillate with a periodicity as given by the

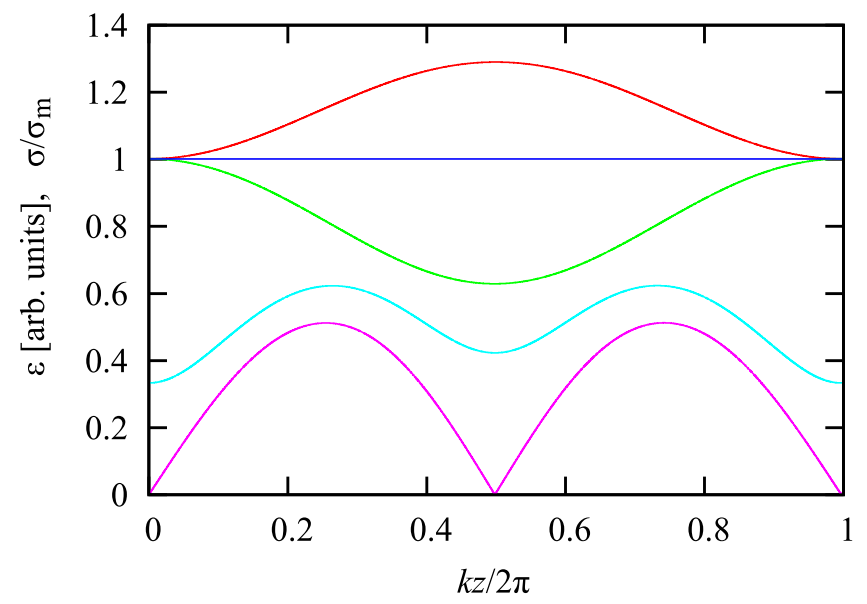

FIG. 10. Example for an emittance oscillation in a constant focusing channel. The average beam size (blue curve) is matched according to Eq. (21). It stays hence constant, while two slices (red and green curves) in the beam and hence also the beam emittance (cyan curve) oscillate due to the difference in the perveance. After half a period, a local emittance minimum is reached. Here the emittance is given by the beam size mismatch [cf. Eq. (16)]; only when the slice emittance is ignored (magenta curve) is a full emittance compensation already reached at this point. wave number [Eq. (23)], and the phase space will resume its initial state after each period. When the slices overlap in phase space at the injection into the solenoid channel, the emittance will hence be compensated again after one period of the oscillation, as illustrated by the example shown in Fig. 10. Note that the emittance after half a period reaches a local minimum, but it is not fully compensated due to the beam size mismatch Eq. (16). This characteristic is quite general and can be observed, for example, also in periodic focusing channels.

It is conceivable to choose other initial state conditions of the internal structure in phase space which fit to the periodic solution to achieve a periodic emittance compensation in the focusing channel. Our ability to control the internal structure in phase space is, however, limited, and a full emittance compensation is not guaranteed.

The general solution for an acceleration section without space charge [Eq. (20) with $P=0$ ] is $[13,16]$

$$
\begin{aligned}
\sigma^{2}= & \sigma_{i}^{2} \cos ^{2} \theta+\frac{2 \gamma_{i}}{\sqrt{K}} \sigma_{i} \sigma_{i}^{\prime} \cos \theta \sin \theta \\
& +\frac{\gamma_{i}^{2}}{K}\left(\frac{\varepsilon_{n}^{2}}{\sigma_{i}^{2}}+\sigma_{i}^{\prime 2}\right) \sin ^{2} \theta,
\end{aligned}
$$

where the index $i$ indicates initial conditions and $\theta$ is given by

$$
\theta=\int \frac{\sqrt{K}}{\gamma} d z=\frac{\sqrt{K}}{\gamma^{\prime}} \ln \left(\frac{\gamma_{i}+\gamma^{\prime} z}{\gamma_{i}}\right)
$$

For $\sigma_{i}^{\prime}=0$ and $\sigma_{i}^{2}=\sigma_{m}^{2}=\frac{\varepsilon_{n}}{\sqrt{K}}$ [cf. Eq. (21) with $K_{s}=0$ ] leads Eq. (24) again to a constant beam size; i.e., the action of the beam emittance and the focusing term are balanced.

For a small mismatch relative to the matched beam condition, Eq. (24) can be rewritten in the form

$$
\sigma \simeq \sigma_{m}+\Delta \sigma \cos 2 \theta+\frac{1}{2} \Delta \sigma^{\prime} \sin 2 \theta
$$

Without an external solenoid field and for $\sin \phi=1$ (on-crest acceleration), $\sqrt{K}$ reduces to $\gamma^{\prime} / 2 \sqrt{2}$. For parameters which are typical for the first acceleration section in a split photoinjector as $\gamma_{i}=10, \gamma^{\prime}=40$, i.e., $20 \mathrm{MV} / \mathrm{m}$ accelerating gradient, $2 \theta=\pi$ is reached after a distance of $21 \mathrm{~m}$, while it would take nearly $2 \mathrm{~km}$ to complete the first period. The oscillation is not damped but strongly stretched. In terms of the energy gain $\frac{\gamma_{i}+\gamma^{\prime} z}{\gamma_{i}}$, the first period is completed when the energy is increased by a factor $\exp (2 \sqrt{2} \pi)=7228$. Adding solenoids reduces the length scale but for technically feasible fields not in a fundamental manner. In a realistic setup with a finite length of the accelerating structures and other interruptions, we will therefore not be able to observe more than a small fraction of a period rather than an oscillation. 
Balancing the focusing term with the defocusing space charge term leads to a solution of Eq. (20) for the case $\varepsilon_{n}=0[6]$.

In order to fulfill the condition

$$
\frac{K}{\gamma^{2}} \sigma=\frac{K_{s}}{\gamma^{3} \sigma},
$$

the beam size has to shrink according to

$$
\sigma=\sigma_{i} \sqrt{\frac{\gamma_{i}}{\gamma}}
$$

and thus

$$
\begin{aligned}
\sigma^{\prime} & =-\frac{\gamma^{\prime}}{2 \gamma} \sigma, \\
\sigma^{\prime \prime} & =\frac{3 \gamma^{\prime 2}}{4 \gamma^{2}} \sigma .
\end{aligned}
$$

Introducing Eqs. (28) and (29) into Eq. (20) with $\varepsilon_{n}=0$ leads for $B_{z}=0$ and $\sin \phi=1$ to

$$
\gamma^{\prime}=\frac{2}{\sigma_{i}} \sqrt{\frac{2 K_{s}}{3 \gamma_{i}}} .
$$

Note that the required initial beam divergence $\sigma_{i}^{\prime}=-\frac{\gamma^{\prime}}{2 \gamma_{i}} \sigma_{i}$ is just introduced by the entrance kick of a cavity when the incoming divergence is zero [13]. Thus, if we adjust the accelerating gradient in accordance to Eq. (30) and match the initial beam divergence outside of the cavity to zero, the beam will travel on the so-called invariant envelope [6]; i.e., the beam size will shrink proportional to $\sqrt{\gamma_{i} / \gamma}$.

The space charge force, however, turns out to be only partially compensated by the ponderomotive focusing force of the rf. A full compensation would require an acceleration which is larger by a factor $\sqrt{3}$ as can be seen by a direct comparison of the engaged forces. The beam envelope is hence determined by the work which the convergent beam has to perform against the partially compensated space charge field and does not correspond to a static equilibrium as in the case of the classical Brillouin flow.

A small mismatch relative to the matched beam conditions leads again to a solution as described by Eq. (26), where now $\sigma_{m}$ is given by $\sigma_{i} \sqrt{\gamma_{i} / \gamma}$ [6], while $\theta$ [Eq. (25)] depends only on the focusing force and thus does not change.

The function of the first accelerating section in a split photoinjector is to convey a space-charge-dominated beam into an emittance-dominated beam while concluding the emittance compensation process in a way that the emittance minimum is reached when the beam becomes fully emittance dominated. The transition from space-charge- to emittance-dominated dynamics takes place over a much

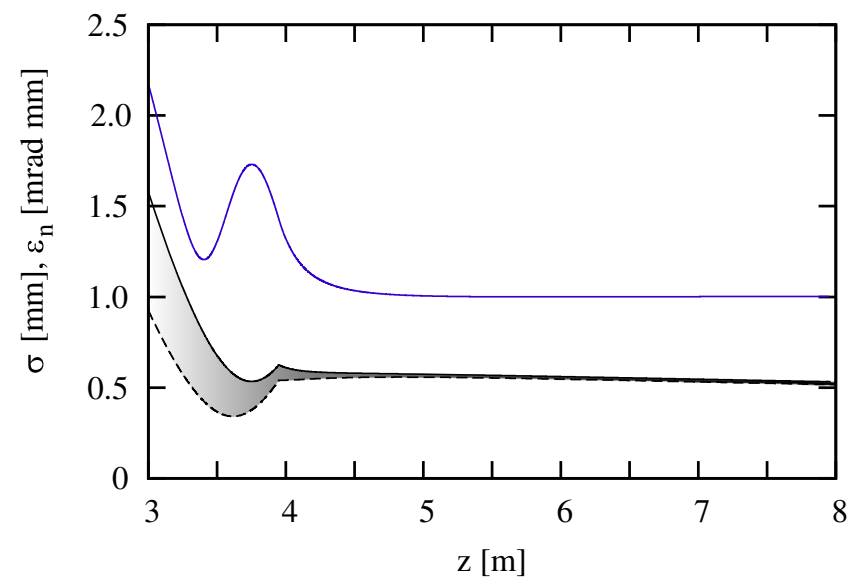

FIG. 11. Emittance (blue line) and slice size (high perveance, solid line; low perveance, broken line) in an accelerating section with $\gamma^{\prime}=41$. The development between $z=0$ and $3 \mathrm{~m}$ is shown in Figs. 3-9. The starting position of the accelerating section is at $z=3.9 \mathrm{~m}$.

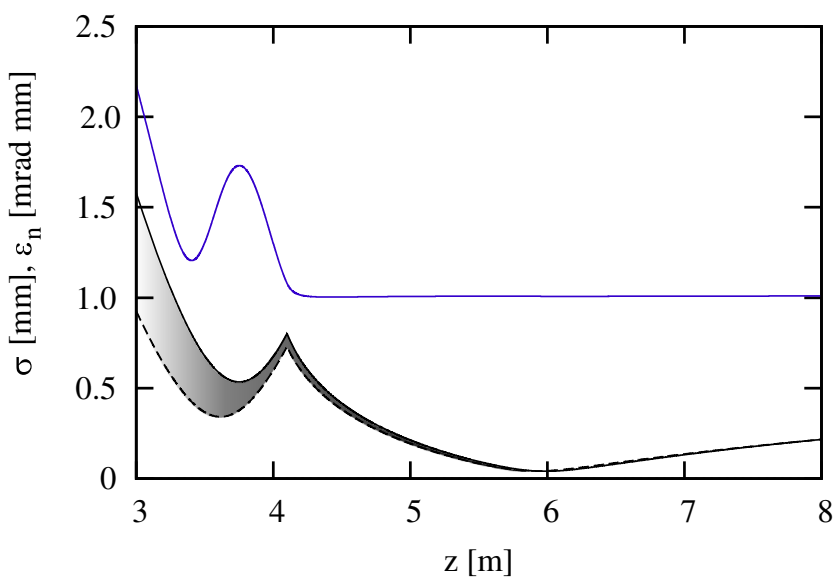

FIG. 12. Emittance (blue line) and slice size (high perveance, solid line; low perveance, broken line) in an accelerating section with $\gamma^{\prime}=82$. The development between $z=0$ and $3 \mathrm{~m}$ is shown in Figs. 3-9. The starting position of the accelerating section is at $z=4.1 \mathrm{~m}$.

shorter distance than the periodicity of an emittance oscillation in a cavity channel. Thus, we do not have to rely on the dynamics of the invariant envelope.

A proper continuation of the emittance compensation process in the accelerating section can be achieved for the gradient suggested by the invariant envelope condition but also for other gradients within a broad parameter range as shown in Figs. 11 and 12. For these calculations, the position of the accelerating section has been varied to minimize the final emittance (for a fixed strength of the focusing element in the upstream drift). For the beam size minimum, i.e., $\sigma^{\prime}=0$, Eq. (30) yields $\gamma^{\prime}=50$. However, when the acceleration starts at the position of the beam size minimum $(z=3.7 \mathrm{~m})$, the gradient has to be reduced to 
$\gamma^{\prime}=29.8$ to achieve the best emittance compensation. For higher gradients, the acceleration section has to be shifted downstream, i.e., closer to the second emittance minimum in order to minimize the final emittance. This is also found in tracking simulations; see, for example, Ref. [17]. The plots show examples for $\gamma^{\prime}=41$ and $\gamma^{\prime}=82$. In all cases, the final emittance is practically given by the slice emittance; i.e., the compensation is nearly perfect (there are marginal differences which are smaller for the higher gradients), but in no case is the beam matched to all invariant envelope parameters.

\section{DISCUSSION OF SIMULATION RESULTS}

The considerations of the previous sections can help to guide the numerical optimization of an injector and to interpret numerical or experimental results, but they do not

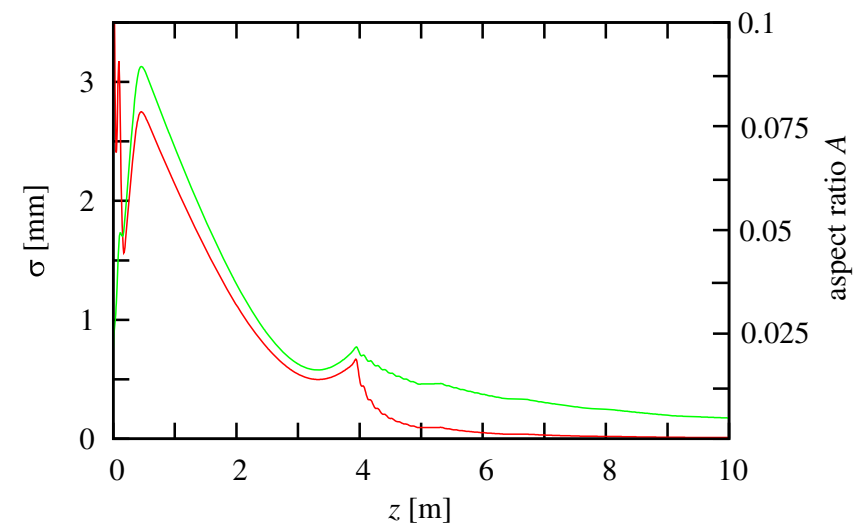

FIG. 13. Transverse beam size (green line) and aspect ratio (red line) in the injector. The maximum beam size occurs inside the solenoid at $0.4 \mathrm{~m}$, and a second, local maximum occurs at the entrance of the cavity section at $\sim 4 \mathrm{~m}$. The beam size minimum at $3.3 \mathrm{~m}$ is close to the local emittance maximum. The effect of overfocused particles onto the beam size is negligible.

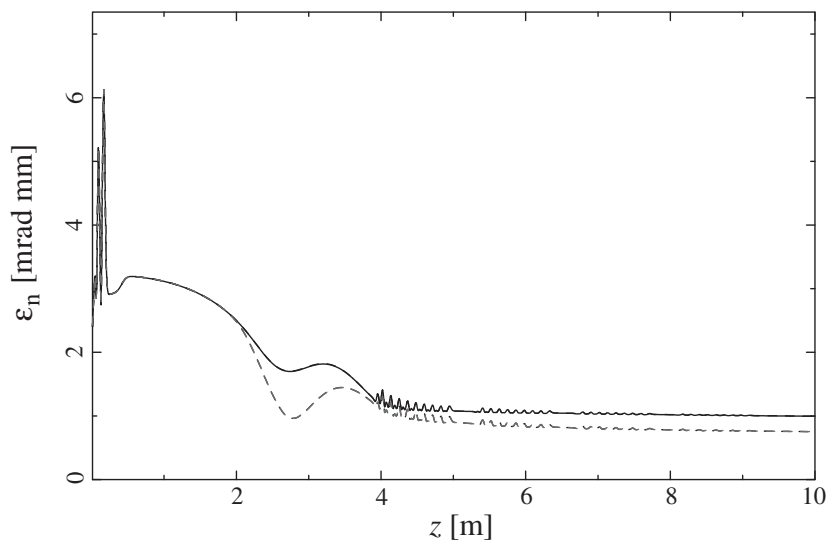

FIG. 14. Development of the projected transverse emittance in the injector. The solid line shows a standard emittance calculation, while in the calculation shown as a dashed line overfocused particles have been ignored. capture the complete dynamics in a photoinjector. In the following, simulation results will be discussed in view of the above developed theory with a focus on aspects which are not described by an envelope model. Different analysis tools which can be used in a simulation work will be presented.

The basic layout of the example injector follows the design of the photoinjector for the European XFEL project [18]. The electrons are generated in a $1 \frac{1}{2}$ cell rf gun operating at $1.3 \mathrm{GHz}$ at a gradient at the cathode of $60 \mathrm{MV} / \mathrm{m}$. A solenoid located $0.4 \mathrm{~m}$ downstream of the cathode focuses the beam, which is then matched into a section of four TESLA-type cavities operating at an accelerating gradient of $21 \mathrm{MV} / \mathrm{m}$ $\left(\gamma^{\prime}=41\right)$. The entrance position of the cavity section, $4 \mathrm{~m}$ downstream of the cathode, has been optimized so as to optimize the transverse emittance at the end of the cavity string. The simulations have been performed with the program ASTRA [19].

Figure 13 shows the development of the beam size and the aspect ratio along the injector, and Fig. 14 the corresponding development of the projected beam emittance. The round beam is launched with an rms spot size of $0.75 \mathrm{~mm}$ and an initial emittance of $\varepsilon_{n}=0.55 \mathrm{mrad} \mathrm{mm}$ at the cathode. A cylindrical charge distribution of $20 \mathrm{ps}$ FWHM length with 2 ps rise or fall time and $1 \mathrm{nC}$ charge is assumed. Both the spot size and the beam emittance increase rapidly up to the center of the solenoid $(z=0.4 \mathrm{~m})$. During the emission, the aspect ratio decreases from infinity (zero charge) up to 2 when the emission is completed. Within the gun cavity, it decreases further, both, due to the increasing beam energy and the increasing bunch length. In the drift, the aspect ratio follows the beam size development and reaches values between 0.075 and 0.01 . Thus, the variation of the space charge force inside the bunch is already reduced close to the

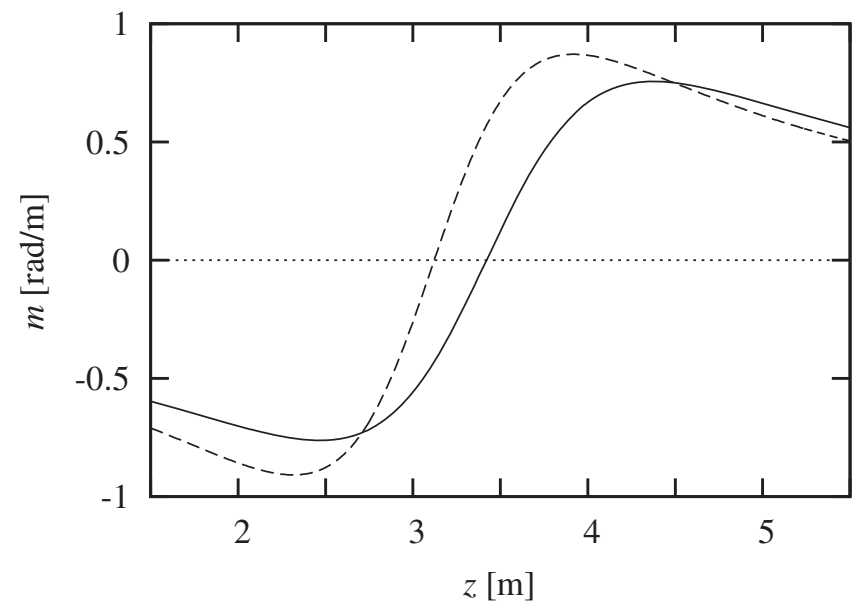

FIG. 15. Development of the slopes of two slices in the drift behind the solenoid. One slice is in the center (solid line), and the other in the head of the bunch (broken line). No acceleration is applied in this case. 

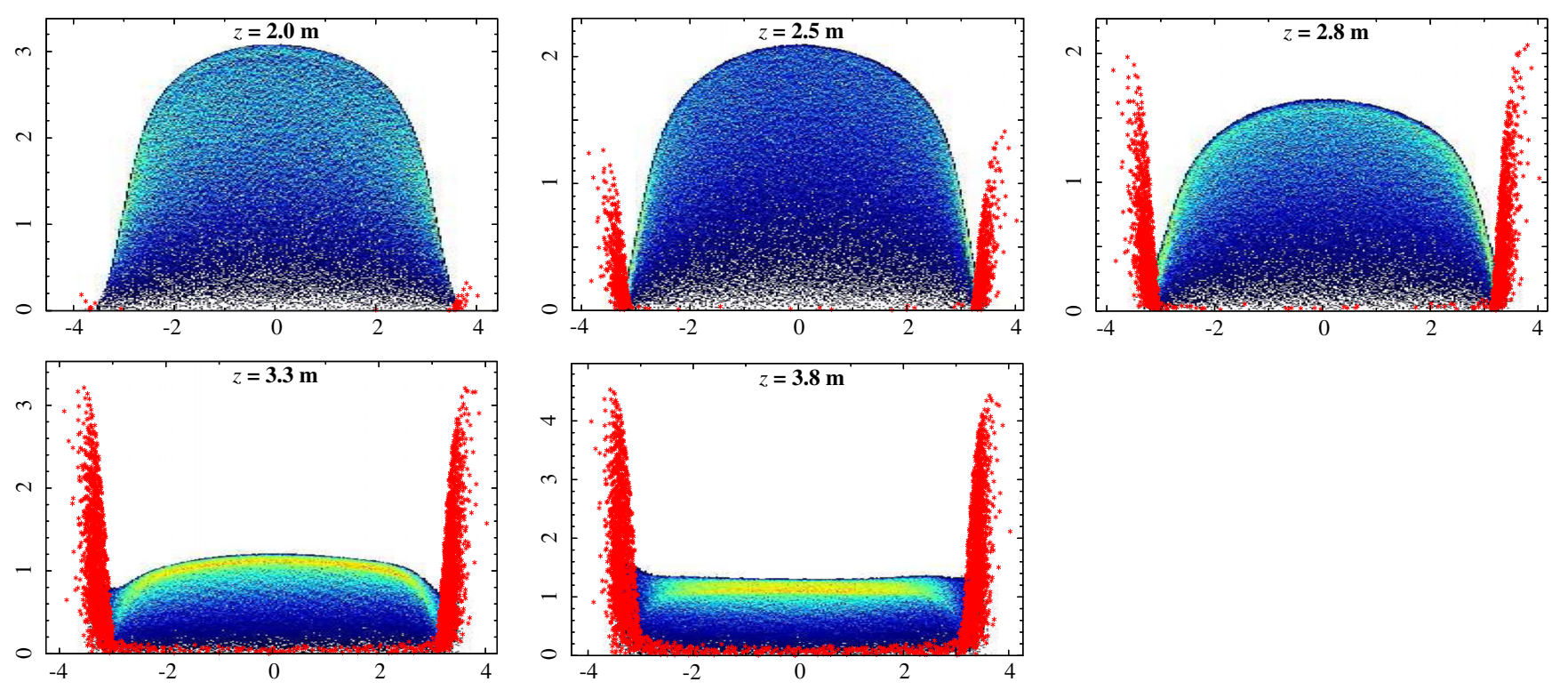

FIG. 16. Particle distribution (radius versus longitudinal position $\zeta$ in millimeters) in the vicinity of the beam size focus at $z=2.0,2.5$, 2.8, 3.3, and $3.8 \mathrm{~m}$. Particles which have been detected as overfocused-plotted as red stars on top of a density map of the not overfocused particles-spill out in the head and tail of the bunch. In total, $1.8 \%$ of the particles are declared as overfocused.

beam size minimum, except for a very pronounced falloff at the head and the tail; cf. Fig. 1.

The emittance development shows in the very beginning the oscillations induced by the rf focusing. In the drift following the solenoid, the emittance decreases similarly as in Fig. 9. In Fig. 15, the slopes of two slices-one in the center, the other in the head of the bunch-are presented. For this calculation, no accelerating cavity is taken into account. The two emittance minima are reached in the vicinity of the crossings of the two lines at $\sim 2.8 \mathrm{~m}$ and $\sim 4.5 \mathrm{~m}$. The final emittance (Fig. 14) is minimized when the beam is divergent at the beginning of the acceleration section (Fig. 13) comparable to Fig. 11. The emittance converges smoothly with small oscillations introduced by the rf focusing to its final value, while the aspect ratio shrinks rapidly due to the acceleration.

The two lines in Fig. 14 present two variants for the emittance calculation. The solid line shows a standard calculation for all particles, while for the calculation shown as a dashed line overfocused particles have been ignored. The difference is explained in the following and illustrated in Fig. 16.

Because of the scaling of the space charge force as $1 / R^{2}$ [Eq. (5)], particles need to work against an increasing field when the beam is focused, so that they lose transverse momentum before they are reflected back without crossing the central axis of the beam. Ideally, particles stay on their relative transverse position within a bunch. This ideal case assumes, however, a stationary particle distribution, which is not valid for a space-charge-dominated beam, as illustrated in Fig. 16. The original cylindrical distribution assumes an ellipsoidal form in the drift up to the focus.
Because of the falloff of the space charge field in the head and the tail of the bunch, particles are here not sufficiently defocused by the space charge field. They move faster toward the beam axis than the beam radius shrinks, and, thus, they can reach the low field region near the beam axis with sufficient momentum to pass through. This process cannot be described in a slice envelope model, because the space charge force scales with the average bunch radius and not with the local slice radius. After passing the beam axis, the particles are accelerated again by the space charge field. Since the beam size is still shrinking, the space charge field is higher during the acceleration phase than it was during the deceleration phase; i.e., in total, the overfocused particles gain transverse momentum from the space charge field. It is this asymmetry in the particle motion which leads to the significant emittance contribution of overfocused particles.

In the simulation, a simple algorithm declares particles as overfocused when they move diagonally from one quadrant of the Cartesian space coordinate system into another. The algorithm is active only in the drift between the exit of the solenoid and the entrance of the cavity string. The majority of particles found with this method are located in the head and the tail of the bunch as shown in Fig. 16. Only a small fraction of about $0.2 \%$ of the total number of particles are distributed over the whole length but close to the transverse center of the bunch. These particles cross over due to the random motion described by the beam emittance. Since overfocused particles are not sufficiently defocused by the space charge field, the crossover appears already in front of the focus of the full beam, mainly in the range from $z=2$ to $2.5 \mathrm{~m}$. 
Figure 14 illustrates the effect of the overfocused particles onto the beam emittance, for which the relative orientation of the two particle ensembles in phase spaceoverfocused and not overfocused-is of relevance. At the first emittance minimum, the main beam is still convergent, while the overfocused ensemble is divergent, so that the total emittance is strongly influenced. The first emittance minimum is flattened nearly to a plateau in the standard calculation, while it shows up as a pronounced minimum in the calculation without the overfocused particles. Later, both ensembles are divergent, so that the effect onto the total emittance is smaller. Ignoring the overfocused particles, a final emittance of $0.75 \mathrm{mrad} \mathrm{mm}$ is reached, while it is $1.1 \mathrm{mrad} \mathrm{mm}$ for the complete particle ensemble. The mismatch of the two ensembles in this example is relatively large, because a relatively high gradient has been chosen in the cavity string section. A better matching between overfocused and not overfocused particles can be reached at a lower gradient.

In order to illustrate the influence of the correlated phase space distortions onto the emittance, it is possible to remove correlations in phase space for the emittance calculation. With the standard emittance formula, only linear correlations in the transverse coordinates $x-p_{x}$ and $y-p_{y}$ are removed, respectively. This approach can be extended by fitting a plane to the $x-p_{x}-\zeta$ coordinates and subtracting the correlations from the particle momenta as

$$
\hat{p}_{x, i}=p_{x, i}-C_{0}-C_{1} x_{i}-C_{2} x_{i} \zeta_{i}-C_{3} x_{i} \zeta_{i}^{2}
$$

(The equivalent calculation is performed for the orthogonal plane.)

$x_{i}, p_{x, i}$, and $\zeta_{i}$ are the initial coordinates of the $i$ th particle. The fit coefficients $C_{0}-C_{3}$ describe a curved plane in the $x-p_{x}-\zeta$ space. $C_{0}$ corresponds to an offset and $C_{1}$ to the average liner correlation as in the standard emittance calculation, $C_{2}$ describes a linear correlation in $\zeta$ as it can

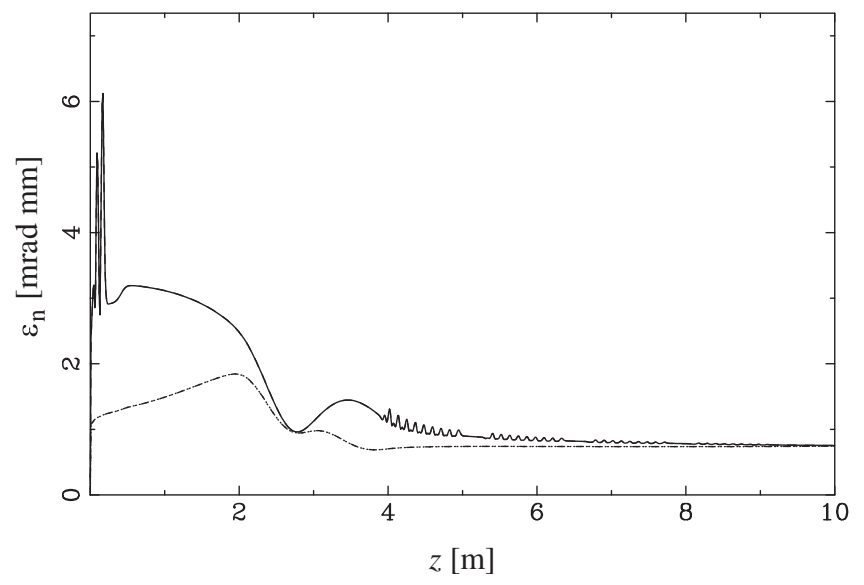

FIG. 17. Comparison of the standard emittance (solid line) and the reduced emittance (dashed line) along the injector beam line.

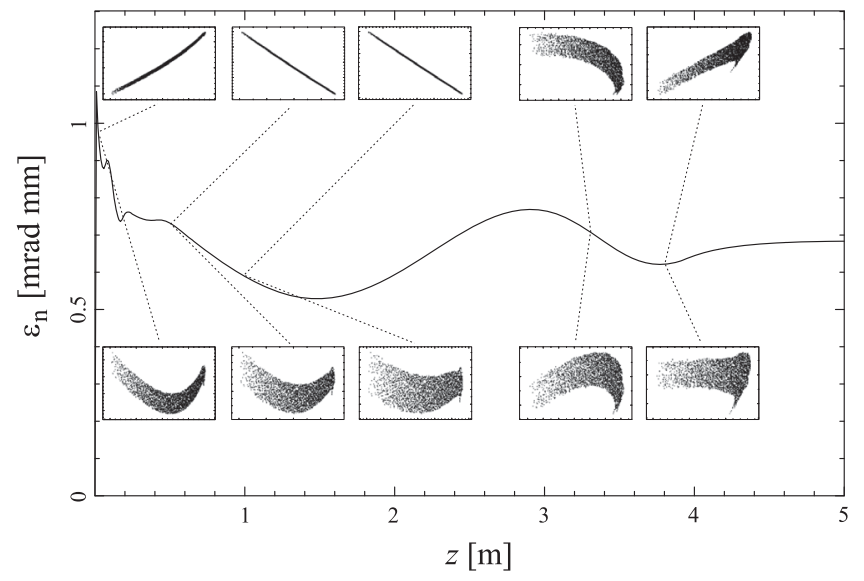

FIG. 18. Emittance of a $90 \mu \mathrm{m}$ long slice in the center of the bunch along the beam line. Correlations with the longitudinal coordinate are not significant due to the short length of the slice. The insets depict the radial phase space $\left(r-r^{\prime}\right)$ at $z=0.01,0.5$, $1.0,3.3$, and $3.8 \mathrm{~m}$. The upper row shows the convergence or divergence of the beam, respectively. For the bottom row, the linear correlation has been removed to pronounce the curvature.

be introduced, e.g., by the rf focusing, and $C_{3}$ describes a quadratic correlation in the longitudinal coordinate as it is introduced by space charge forces. After subtracting the correlations, the so-called reduced emittance follows from a multiplication of the rms values as $\varepsilon_{\text {red }}=\sigma_{x} \sigma_{\hat{p}_{x}}$.

The symmetric part of the space charge force is only approximately described by the quadratic term. A further expansion of Eq. (31) to even higher orders is, however, in general not necessary.

The reduced emittance is compared to the standard emittance in Fig. 17. For both calculations, the overfocused particles have been ignored. The reduced emittance shows much smaller excursions than the standard emittance. The influence of the beam size mismatch, which is not removed by this method, becomes especially visible near $2 \mathrm{~m}$. At the first emittance minimum, the reduced and the standard emittance are equal, since here also the standard emittance is increased only by the beam size mismatch. The difference between the absolute minimum of the reduced emittance $(0.70 \mathrm{mrad} \mathrm{mm}$ at $\sim 3.8 \mathrm{~m})$ and the end value $(0.75 \mathrm{mrad} \mathrm{mm})$ indicates a small beam size mismatch of the final emittance. Thus, correlations in the orientation of slices are very well compensated; still, the final emittance is larger than the initial beam emittance of $0.55 \mathrm{mrad} \mathrm{mm}$.

This discrepancy between the initial and final emittance is related to the dynamics in close vicinity of the cathode [20] and shows up in the (central) slice emittance as presented in Fig. 18.

For this plot, the emittance of a subensemble of particles, which forms at the end of the beam line, a slice of $90 \mu \mathrm{m}$ length in the longitudinal center of the bunch is calculated. Because of differences in the effective accelerating fields near the cathode and differences in the path length, the 
longitudinal position of particles in the radial border changes relative to the position of particles in the center of the distribution, while the beam travels through the beam line. Thus, the subensemble starts as a curved, dish-shaped object and straightens up toward the end of the beam line. The longitudinal dynamics introduces energy-position correlations, which influence also the transverse divergence angle but appear to be insignificant as compared to the direct space charge effects.

The nonlinear transverse space charge field of bunches with a large aspect ratio (cf. Fig. 2) drives the emittance of the slice right at the cathode from $0.55 \mathrm{mrad} \mathrm{mm}$ to about $1.0 \mathrm{mrad} \mathrm{mm}$. At $z=1 \mathrm{~cm}$, the beam is highly divergent and the divergence increases with increasing radius (first inset in Fig. 18). This results in a dilution of the space charge density at the rim of the distribution which leads in the following to a reduction of the space charge field in this area. Particles in the diluted area will hence experience a reduced space charge field - as compared to the linear field of a uniform distribution with $A<1$ - so that the curvature in phase space reduces again. The effect is self-linearizing; however, the dynamics does not stop when the emittance minimum is recovered at $z=1.5 \mathrm{~m}$ but continues to bend the phase space so that the slice emittance increases again. The process repeats similarly; however, the phase space tends to fold over, so that the minimal emittance cannot be recovered again.

The observed development varies in its details with the applied focusing strength of the solenoid, which needs to be optimized for the emittance compensation process. Moreover, it is different for different longitudinal positions within the bunch, i.e., for different slices, because the induced nonlinearity changes during the emission of the bunch.

\section{SUMMARY}

The slice envelope model discussed in this paper is based on an analytic solution of the beam envelope equation in a drift and takes both the space charge term and the emittance term into account. This allows us to describe the emittance development including the emittance contribution originating from a beam size mismatch. The appearance of two emittance minima close to the beam size minimum is related to the difference of the slopes of the phase space ellipses in the divergent state, before the beam is focused by a solenoid lens. The smaller beam size mismatch at the second emittance minimum is the result of the shifted focus positions of slices with different perveance.

The discussion of the emittance compensation in focusing channels revealed that only every second minimum leads to a complete emittance compensation, while the local minima in between are somewhat larger due to the beam size mismatch. When the final emittance is minimized, the matching into the booster cavity does not follow the invariant envelope conditions in a split photoinjector design.
In the final section, numerical results are compared to the slice envelope model. The final emittance of the complete particle ensemble is influenced by the matching between the main part of the bunch and a small fraction of overfocused particles which are generated already before the beam size minimum is reached. Another factor which is not captured by the slice model is the emittance growth due to space charge field nonlinearities near the cathode. The induced curvature in the transverse phase space has a tendency to self-linearize; the process is, however, also influenced by the focusing conditions in the injector beam line. The ideal parameter setting which minimizes the final emittance represents, hence, a compromise between the different emittance contributions which cannot be optimized independently of each other.

\section{ACKNOWLEDGMENTS}

I thank B. Zeitler (UHH/CFEL) for proofreading and useful suggestions.

\section{APPENDIX: CONVERGENCE CRITERION FOR THE POLYNOMIAL SOLUTION}

When more coefficients are taken into account in the generating Eq. (8), they show up only in terms proportional to orders higher than $z^{4}$ in the extended Eq. (9), and thus they can be determined by successively setting terms with increasing order in $z$ equal to zero. This yields for the next two orders

$$
\begin{aligned}
& c=\left(\frac{b P}{4}-3 a b\right) /\left(15 \sigma_{0}^{2}\right), \\
& d=\left(\frac{c P}{4}-2 b^{2}-10 a c\right) /\left(28 \sigma_{0}^{2}\right) .
\end{aligned}
$$

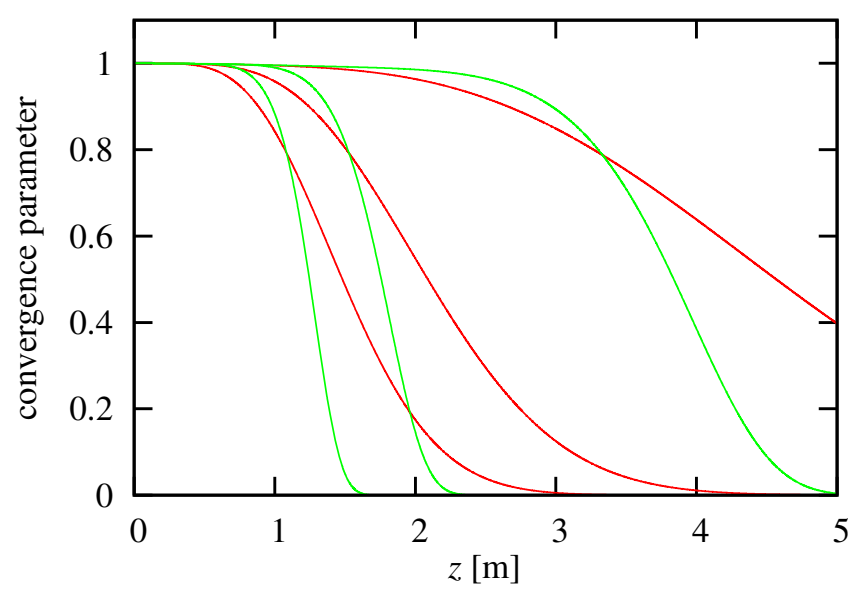

FIG. 19. Development of the convergence parameter Eq. (A3) of the polynomial series in a drift. Fourth-order approximation (red curves) and eighth-order approximation (green curves). Beam parameters: $\gamma=10, \varepsilon_{n} / \gamma=10^{-7}$, and $P=(10,5,1) \times 10^{-6}$. 
It is also possible to set up the equation for the asymmetric case where $z=0$ is not a focus; see [21] for details.

Note that, in the limiting case $\frac{\varepsilon^{2}}{\sigma_{0}^{2}}>\frac{P}{4}, a$ reduces to $\frac{\varepsilon^{2}}{\sigma_{0}^{2}}$; all following coefficients remain, however, finite as long as $P$ is not exactly zero. Thus, space charge forces can be ignored only if in addition to $\frac{\varepsilon^{2}}{\sigma_{0}^{2}}>\frac{P}{4}$ the condition $\sigma_{0}^{2}+\frac{\varepsilon^{2}}{\sigma_{0}^{2}} z^{2}>b z^{4}$ is fulfilled. The emittance-dominated regime appears in this sense to be fundamentally limited to small beam sizes. The argument is, however, alleviated by the drop of the perveance with an increasing aspect ratio as discussed in Sec. II.

The envelope equation without an emittance term can be directly integrated to define a convergence parameter. Form $\sigma^{\prime \prime}=\frac{P}{4 \sigma}$ follows [11]:

$$
\sigma^{\prime 2}=\frac{P}{2} \ln \frac{\sigma}{\sigma_{0}}
$$

and thus

$$
\frac{\sigma}{\sigma_{0}} / \exp \left(\frac{2 \sigma^{\prime 2}}{P}\right)=1
$$

In Fig. 19, the convergence parameter as defined by Eq. (A3) is compared for the fourth-order (red curves) and the eighth-order (green curves) approximation of the polynomial solution for different values of the beam perveance. When the convergence parameter gets significantly smaller than 1 , the polynomial approximation deviates from numerical results; cf. Fig. 3. As example, at a convergence parameter of 0.4 , the ratio $\sigma / \sigma_{0} \approx 3.3$ and the polynomial solution deviates by about $10 \%$ from the numerical result in the fourth-order approximation.

While the onset of the falloff of the convergence parameter is shifting towards larger $z$, it is also getting steeper with the increasing number of orders and, thus, the green lines cross the corresponding red lines in Fig. 19 at a convergence parameter of $\sim 0.8$. The approximate results are therefore improved only up to the corresponding positions in $z$, while they deviate even stronger for larger $z$. To shift the falloff to significantly higher values in $z$ would require one to take many more orders in the polynomial expansion into account [21].

[1] C. H. Lee, P. E. Oettinger, E. Pugh, R. Klinkowstein, J. Jacob, J. S. Fraser, and R. L. Sheffield, Electron emission of over $200 \mathrm{~A} / \mathrm{cm}^{2}$ from a pulsed-laser irradiated photocathode, IEEE Trans. Nucl. Sci. 32, 3045 (1985).

[2] J. S. Fraser, R. L. Sheffield, and E. R. Gray, A new highbrightness electron injector for free electron lasers driven by RF linacs, Nucl. Instrum. Methods Phys. Res., Sect. A 250, 71 (1986).

[3] B. E. Carlsten, New photoelectric injector design for the Los Alamos National Laboratory XUV FEL accelerator, Nucl. Instrum. Methods Phys. Res., Sect. A 285, 313 (1989).

[4] M. Ferrario, J. E. Clendenin, D. T. Palmer, J. B. Rosenzweig, and L. Serafini, Report No. SLAC-PUB-8400, 2000.

[5] M. Ferrario et al., Direct Measurement of the Double Emittance Minimum in the Beam Dynamics of the Sparc High-Brightness Photoinjector, Phys. Rev. Lett. 99, 234801 (2007).

[6] L. Serafini and J. B. Rosenzweig, Envelope analysis of intense relativistic quasilaminar beams in rf photoinjectors: A theory of emittance compensation, Phys. Rev. E 55, 7565 (1997).

[7] C.-x. Wang, Hamiltonian analysis of transverse beam dynamics in high-brightness photoinjectors, Phys. Rev. E 74, 046502 (2006); Comment on the invariant-envelope solution in rf photoinjectors, Nucl. Instrum. Methods Phys. Res., Sect. A 557, 94 (2006).

[8] S. V. Miginsky, Emittance compensation of elliptical beams, Nucl. Instrum. Methods Phys. Res., Sect. A 603, 32 (2009).

[9] M. Ferrario, A. Mosnier, L. Serafini, F. Tazzioli, and J. M. Tessier, Multi-bunch energy spread induced by beam loading in a standing wave structure, Part. Accel. 52, 1 (1996).

[10] E. R. Harrison, On the space-charge divergence of an axially symmetric beam, J. Electron. Control 4, 193 (1958).

[11] J. D. Lawson, The Physics of Charged-Particle Beams (Oxford University Press, New York, 1988).

[12] K. Floettmann, rf-induced beam dynamics in rf guns and accelerating cavities, Phys. Rev. ST Accel. Beams 18, 064801 (2015).

[13] S. C. Hartmann and J. B. Rosenzweig, Ponderomotive focusing in axisymmeric rf linacs, Phys. Rev. E 47, 2031 (1993); J. Rosenzweig and L. Serafini, Transverse particle motion in radio-frequency linear accelerators, Phys. Rev. E 49, 1599 (1994).

[14] L. Brillouin, A Theorem of Larmor and Its Importance for Electrons in Magnetic Fields, Phys. Rev. 67, 260 (1945).

[15] M. Reiser, Theory and Design of Charged Particle Beams (Wiley, New York, 1994).

[16] Note that the emittance is not conserved in combined cavity-solenoid sections; for details, see Ref. [12].

[17] S. Bettoni, M. Pedrozzi, and S. Reiche, Low emittance injector design for free electron lasers, Phys. Rev. ST Accel. Beams 18, 123403 (2015).

[18] M. Ferrario, K. Floettmann, B. Grigoryan, T. Limberg, and Ph. Piot, Report No. TESLA-FEL 2001-03, 2001.

[19] K. Floettmann, ASTRA-A space charge tracking algorithm, http://www.desy.de/ mpyflo.

[20] R. Cee, M. Krassilnikov, S. Setzer, T. Weiland, and A. Novokhatski, Detailed numerical studies of space charge effects in an FEL RF gun, Nucl. Instrum. Methods Phys. Res., Sect. A 483, 321 (2002).

[21] M. Hachmann, Diploma thesis, University of Hamburg, 2012. 